\title{
The Integrity of piRNA Clusters is Abolished by Insulators in the Drosophila Germline
}

\author{
Elizaveta Radion ${ }^{1}$, Olesya Sokolova ${ }^{1}$, Sergei Ryazansky ${ }^{1}$ (1) , Pavel A. Komarov 1,2 ${ }^{10}$, \\ Yuri Abramov ${ }^{1}$ and Alla Kalmykova ${ }^{1, *(D)}$ \\ 1 Institute of Molecular Genetics, Russian Academy of Sciences, 123182 Moscow, Russia; \\ sradion-radion.90@mail.ru (R.E.); sokolova@img.ras.ru (O.S.); ryazansky@img.ras.ru (S.R.); \\ pkom94@gmail.com (P.A.K.); abramov75@rambler.ru (Y.A.) \\ 2 Present Address: Friedrich Miescher Institute for Biomedical Research, Maulbeerstrasse 66, \\ 4058 Basel, Switzerland \\ * Correspondence: allakalm@img.ras.ru; Tel.: +7(499)-1960019; Fax: +7(499)-1960221
}

Received: 29 January 2019; Accepted: 6 March 2019; Published: 11 March 2019

\begin{abstract}
Piwi-interacting RNAs (piRNAs) control transposable element (TE) activity in the germline. piRNAs are produced from single-stranded precursors transcribed from distinct genomic loci, enriched by TE fragments and termed piRNA clusters. The specific chromatin organization and transcriptional regulation of Drosophila germline-specific piRNA clusters ensure transcription and processing of piRNA precursors. TEs harbour various regulatory elements that could affect piRNA cluster integrity. One of such elements is the suppressor-of-hairy-wing $(\mathrm{Su}(\mathrm{Hw}))$-mediated insulator, which is harboured in the retrotransposon gypsy. To understand how insulators contribute to piRNA cluster activity, we studied the effects of transgenes containing gypsy insulators on local organization of endogenous piRNA clusters. We show that transgene insertions interfere with piRNA precursor transcription, small RNA production and the formation of piRNA cluster-specific chromatin, a hallmark of which is Rhino, the germline homolog of the heterochromatin protein 1 (HP1). The mutations of $\mathrm{Su}(\mathrm{Hw})$ restored the integrity of piRNA clusters in transgenic strains. Surprisingly, $\mathrm{Su}(\mathrm{Hw})$ depletion enhanced the production of piRNAs by the domesticated telomeric retrotransposon TART, indicating that $\mathrm{Su}(\mathrm{Hw})$-dependent elements protect TART transcripts from piRNA processing machinery in telomeres. A genome-wide analysis revealed that $\mathrm{Su}(\mathrm{Hw})$-binding sites are depleted in endogenous germline piRNA clusters, suggesting that their functional integrity is under strict evolutionary constraints.
\end{abstract}

Keywords: drosophila; retrotransposons; transgene; piRNA cluster; insulator; $\mathrm{Su}(\mathrm{Hw})$; $\mathrm{Rhino;}$ germline; transcription; $\mathrm{HeT}-\mathrm{A}$ and TART telomeric retrotransposons

\section{Introduction}

The Piwi-interacting RNA (piRNA) pathway is an essential mechanism that protects genome integrity by suppressing transposable element (TE) activity in animal gonads [1]. In Drosophila, piRNA precursors are derived from distinct genomic regions termed piRNA clusters, which are enriched in TE fragments [2]. The specific chromatin structure of piRNA clusters ensures the recruitment of the noncanonical transcriptional machinery that drives piRNA precursor expression [3-6]. The chromatin of piRNA clusters is enriched in a common heterochromatic histone mark, trimethylated lysine 9 of histone $\mathrm{H} 3$ (H3K9me3) and by two chromodomain-containing proteins, heterochromatic protein 1 (HP1) and its germline-specific ortholog Rhino (Rhi) [4,7-9]. The protein Maelstrom represses canonical transcription from TEs and neighbouring gene promoters in dual-strand piRNA clusters [10]. Instead, noncanonical convergent transcription from both genomic strands initiated at multiple 
random sites facilitates the transcription of piRNA precursors from dual strand piRNA clusters $[6,7]$. The initiation of such noncanonical transcription within the heterochromatin of piRNA clusters is mediated by the germline-specific TFIIA-L paralog Moonshiner, which forms an alternative RNA Polymerase II preinitiation complex in Rhi-enriched domains [6]. Rhi binding suppresses the splicing of piRNA precursors [11]. In addition, Rhi recruits Cutoff (Cuff) protein [4], which mediates the generation of long read-through transcripts from piRNA clusters by inhibiting termination at poly(A) sites [12]. Finally, the transcription-export (TREX) complex participates in the export of unspliced piRNA precursors from the nucleus to the cytoplasmic piRNA processing machinery $[13,14]$. The Piwi-dependent establishment of piRNA cluster identity occurs during early embryogenesis, which is crucial for TE repression at later developmental stages [9]. The integrity of piRNA clusters is an important factor in antitransposon defence, since the adaptivity of the piRNA system is based on the ability of alien sequences inserted within piRNA clusters to become their integral part and produce cognate piRNAs [15-18].

Insulators and their binding proteins play an essential role in transcription regulation by limiting inappropriate enhancer-promoter interactions of neighbouring genes or by blocking repressive chromatin spreading [19]. Insulators are found in the regulatory regions of genes and at homeotic gene loci and the boundaries of topologically associating domains, TADs [20,21]. Some Drosophila retrotransposons also contain insulators [22-24]. One of the best-characterized TE insulators is located in the regulatory region of the gypsy long terminal repeat (LTR) retrotransposon and contains binding sites for the suppressor of hairy wing $(\mathrm{Su}(\mathrm{Hw}))$ zinc-finger protein [25]. This DNA-binding protein establishes the multicomponent chromatin complex important for transcriptional regulation and germline development $[19,26]$. piRNA clusters contain different TEs, including those demonstrating insulator activity; however, the hierarchical relationship between the chromatin of piRNA clusters and insulator complex formation is not clear. We show here that transgenes bearing $\mathrm{Su}(\mathrm{Hw})$ recognition sites embedded in endogenous pericentromeric and telomeric piRNA clusters interfere with the local transcription of piRNA precursors, production of small RNAs and formation of specific chromatin structure.

\section{Materials and Methods}

\subsection{Drosophila Transgenic Strains}

The transgenic strain KG10047 carrying the insertion of the P\{SUPor-P\} element in the HeT-A 3' UTR was described previously [27]. Transgenic strain KG09351 (Bloomington Drosophila Stock Centre \#16481; the strain was terminated) carries a P\{SUPor-P\} insertion in the $42 A B$ locus at the position 2R:2,160,357 [-] (according to the $\mathrm{dm} 3$ genome assembly). The transgenic strain KG02245 (Bloomington Drosophila Stock Centre \#12975) carries a P\{SUPor-P\} insertion in the 49E locus. Transheterozygous $s u(H w)^{V} / s u(H w)^{f}$ flies were used in the study.

\subsection{Small RNA Library Preparation and Analysis}

Small RNAs 19-29 nt in size from total ovarian RNA extracts were cloned as previously described [18]. The libraries were barcoded according to Illumina TrueSeq Small RNA sample prep kit instructions and submitted for sequencing using the Illumina HiSeq-2000 sequencing system (San Diego, CA, USA). After clipping the Illumina 3'-adapter sequence, small RNA reads that passed quality control and minimal length filter $(>18 \mathrm{nt}$ ) were mapped (allowing 0 mismatches) to the Drosophila melanogaster genome (Apr. 2006, BDGP assembly R5/dm3) or transgenes by bowtie2 [28]. The plotting of size distributions, read coverage and nucleotide biases were performed as described previously [18]. To identify piRNAs (24-29 nt reads) or siRNAs (21 nt reads) derived from TEs and piRNA clusters, small RNA reads were mapped to the canonical sequences of transposable elements (http:/ /www.fruitfly.org/p_disrupt/TE.html) or to the piRNA clusters [2] by bowtie2 [28]. 
Ovarian small RNA-seq data for KG10047;+/+, KG10047;su(Hw) $/ \mathrm{su}(\mathrm{Hw})^{f}, \mathrm{KG09351;+/+ \text {; }}$

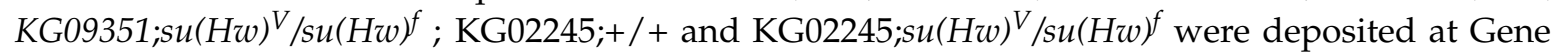
Expression Omnibus (GEO), accession number GSE125173.

\subsection{Chromatin Immunoprecipitation (ChIP)}

ChIP was performed according to the published procedure [7]. Chromatin was immunoprecipitated with the following antibodies: anti-HP1a (C1A9 Developmental Studies Hybridoma Bank, Iowa Sity, IA, USA), anti-trimethyl-histone H3 Lys9 (Millipore, Burlington, MA, USA), Rhi antiserum [29] and anti-Su(Hw) [30]. Primers used in the study are listed in Table S1. Quantitative PCR was conducted with a LightCycler 96 (Roche, Basel, Switzerland). Obtained values were normalized to input and compared with values at rp 49 gene as a control genomic region. Standard error of mean (SEM) of triplicate PCR measurements for three biological replicates was calculated.

\subsection{RT-PCR}

RNA was isolated from the ovaries of three-day-old females. cDNA was synthesized using random hexamers or strand-specific primers and SuperScriptII reverse transcriptase (Life Technologies, Carlsbad, CA, USA). cDNA samples were analysed by real-time quantitative PCR using SYTO-13 dye on a LightCycler96 (Roche, Basel, Switzerland). Values were averaged and normalized to the expression level of the ribosomal protein gene rp49. The primers used are described in Table S1.

\subsection{Motif Finding}

To estimate the frequency of the $\mathrm{Su}(\mathrm{Hw})$ insulator sites, the corresponding PWM profile (MA0533.1 from JASPAR_2016 database) was searched against the dm6 genome assembly or piRNA cluster regions [2] by using fimo 4.11.1 from the MEME suite [31]. The $p$-value $1 \times 10^{-5}$ was used as the threshold level.

\section{Results and Discussion}

\subsection{P\{SUPor-P\} Transgenic Constructs Inserted into piRNA Clusters Do Not Produce piRNAs}

To study how insulators affect piRNA cluster integrity, we used P\{SUPor-P $\}$ transgenic constructs carrying two gypsy insulators and located within endogenous piRNA clusters. KG10047 and KG09351 transgenes were inserted into the 3'UTR of telomeric retrotransposon $\mathrm{HeT}-\mathrm{A}$ and the major pericentromeric piRNA cluster in the $42 A B$ locus, respectively (Figure $1 \mathrm{~A}$ ).

Both integrated loci were previously described as potent piRNA clusters able to adapt new insertions for piRNA production [12,15,16,32]. The euchromatic KG02245 transgene was used as a control. To determine whether the constructs carrying gypsy insulators are able to become a part of piRNA clusters and produce piRNAs, we sequenced small RNAs from the ovaries of the transgenic strains. The mapping of small RNAs to P\{SUPor-P\} revealed a negligible amount of the transgene-derived small RNAs in both cases (Figure 1B). We suggested that $\mathrm{Su}(\mathrm{Hw})$ binding could impede piRNA production and performed ovarian small RNA sequencing of transgenic strains bearing $s u(H w)^{V} / s u(H w)^{f}$ mutations. These mutations cause the loss of $\mathrm{Su}(\mathrm{Hw})$ binding to the gypsy insulator $[33,34]$ and, as we show in the next section, to P\{SUPor-P $\}$ transgene (Figure $2 \mathrm{~B})$. Su(Hw) mutations result in the production of abundant transgenic small RNAs, most of which are 24-29 nt long and demonstrate $5^{\prime}$ terminal uridine bias (1U bias), which is a characteristic of piRNAs (Figure 1B,C). However, we did not find the sense/antisense piRNA pairs overlapping by $10 \mathrm{nt}$, which is a signature of the ping-pong piRNA amplification cycle [2,35] (Figure 1D). This result indicates that primary processing plays a major role in transgenic piRNA production. The most likely explanation is that a low abundance of transgenic transcripts prevents efficient ping-pong amplification. The euchromatic transgene KG02245 (control) produces a negligible amount of small RNAs in wild type and $\mathrm{Su}(\mathrm{Hw})$ mutant backgrounds (Figure 1). 
A
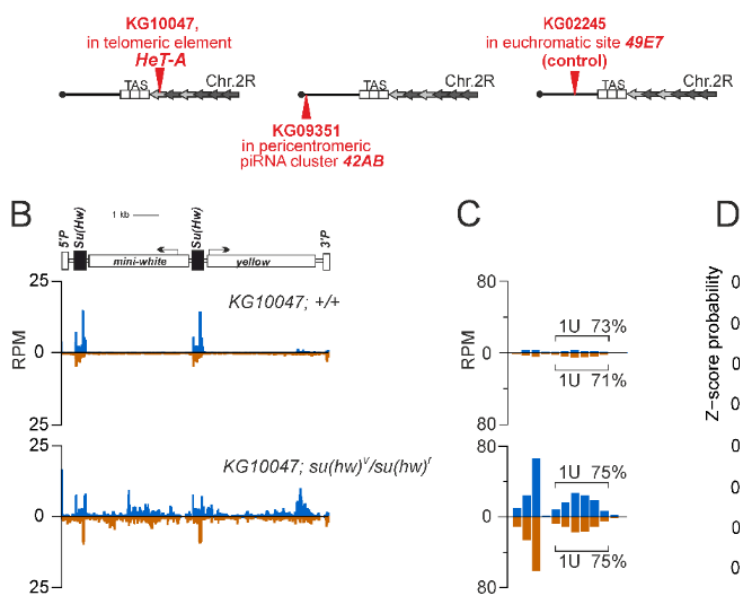

C
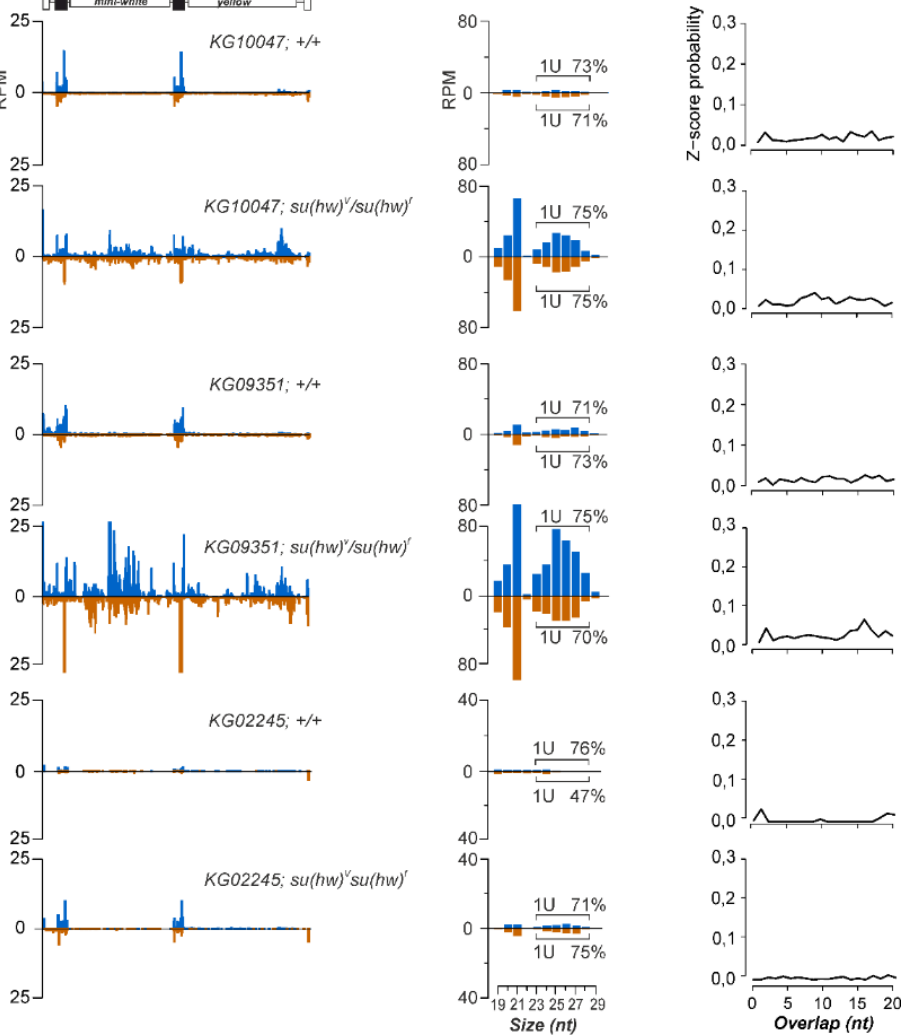

Figure 1. Generation of small RNAs by transgenes containing $\mathrm{Su}(\mathrm{Hw})$-binding sites and located in piRNA clusters. (A) Schematic representation of transgenic insertion sites. Insertion sites of transgenes are indicated as triangles situated either up or below the schemes, which correspond to their genomic orientation. (B) Scheme of SUPor-P construct is shown above. Normalized numbers of small RNAs (19-29 nt, in reads per million, RPM) mapped to transgenic constructs (blue-sense; brown—antisense; no mismatches allowed) in wild type Drosophila ovaries and in $s u(H w)^{V} / s u(H w)^{f}$ mutants. (C) Length distribution of transgenic small RNAs. Percentage of transgenic reads excluded $\mathrm{Su}(\mathrm{Hw})$ sites having $1 \mathrm{U}$ is indicated for each strand (only 24-29-nt reads were considered). (D) Relative frequencies (Z-score) of $5^{\prime}$ overlap for sense and antisense 24-29-nt transgenic piRNAs excluded $\mathrm{Su}(\mathrm{Hw})$ sites (ping-pong signature). 
A

$\mathrm{B}$
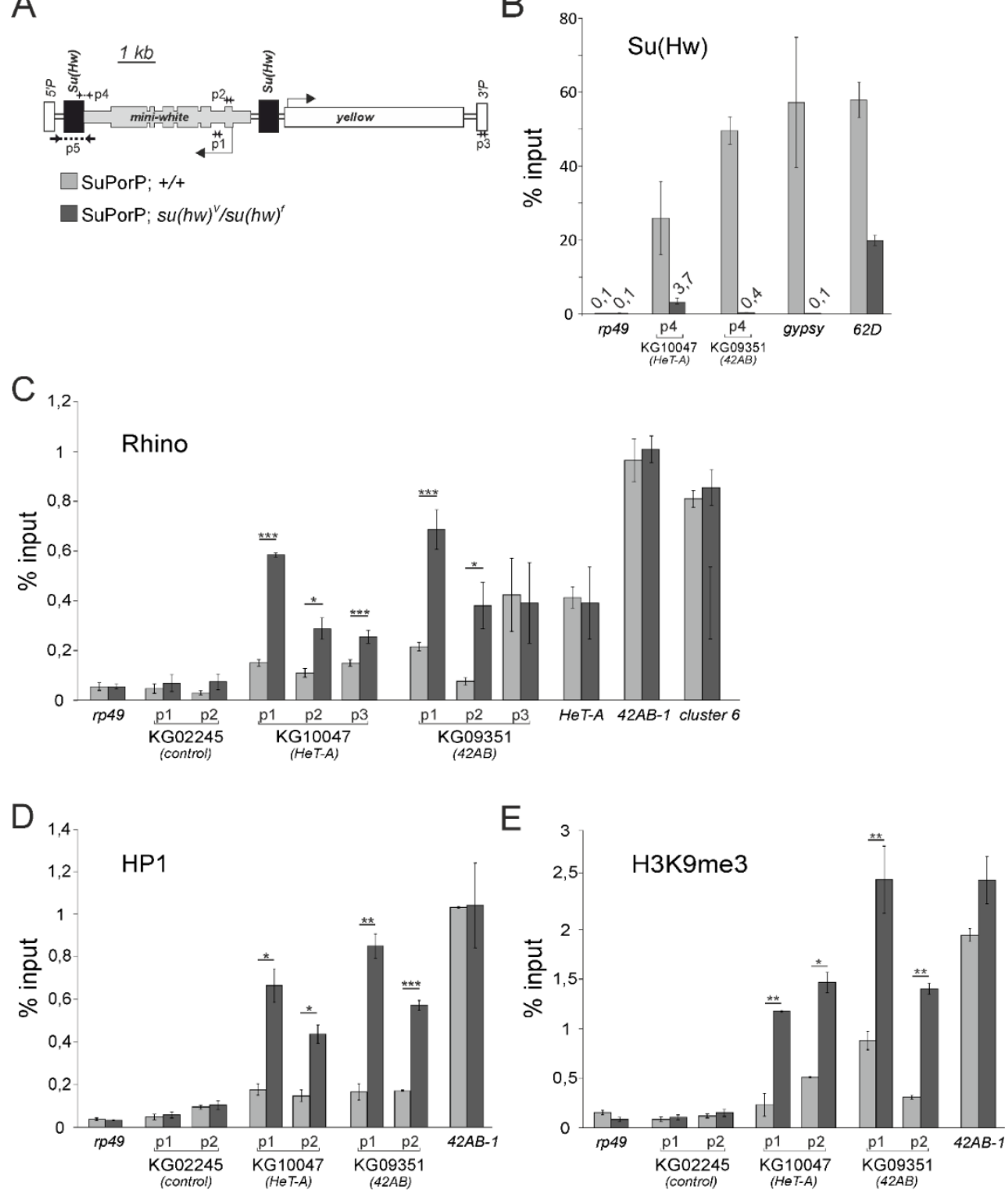

Figure 2. Chromatin components and transcription state of the transgenes containing $\mathrm{Su}(\mathrm{Hw})$ sites and located within piRNA clusters. (A) Schematic representation of the P\{SUPor-P\} transgene and the positions of the primers used in ChIP (p1, p2, p3, p4) and RT-PCR (p1, p3, p5) are shown. (B) Su(Hw) binds $\mathrm{P}\{\mathrm{SUPor}-\mathrm{P}\}$ transgenes in $\mathrm{Su}(\mathrm{Hw})$ wild type but not in mutant ovaries. The $r p 49$ is used as a control devoid of $\mathrm{Su}(\mathrm{Hw})$ binding sites. As expected, the gypsy insulator lost $\mathrm{Su}(\mathrm{Hw})$ binding in the $s u(H w)^{V} / s u(H w)^{f}$ background but the insulator in the $62 \mathrm{D}$ locus retained $\mathrm{Su}(\mathrm{Hw})$ association $[33,34]$. Mean values are indicated only for low levels of $\mathrm{Su}(\mathrm{Hw})$ binding. (C-E) Rhi (C), HP1 (D) and H3K9me3 (E) occupancies at $\mathrm{P}\{\mathrm{SUP}$ - $\mathrm{P}\}$ transgenes in wild type and $s u(H w)^{V} / s u(H w)^{f}$ mutants were estimated by ChIP-qPCR using indicated primers (p1, p2, p3). The regions of the endogenous $42 A B$ and \#6 piRNA clusters and telomeric retrotransposon $H e T-A$ are enriched by all studied chromatin components and used as positive controls. $r p 49$ is used as a negative control. Asterisks indicate statistically significant differences in chromatin protein enrichments between wild type and $s u(H w)^{V} / s u(H w)^{f}$ mutants ( ${ }^{*} p<0.05$ to $0.01,{ }^{* *} p<0.01$ to $0.001,{ }^{* * *} p<0.001$, unpaired $t$-test). Error bars represent SEM of 3 biological replicate experiments. For HP1 and H3K9me3 binding to KG02245, the error bars represent $\mathrm{SD}$ of three technical replicates.

Interestingly, a significant fraction of the small RNAs produced by both $\mathrm{P}\{\mathrm{SUP}$ Por-P $\}$ transgenes in $\mathrm{Su}(\mathrm{Hw})$ mutants are 21-nt endogenous small interfering RNAs (endo-siRNAs) (Figure 1C). Indeed, it has been reported that endogenous and transgenic piRNA clusters also produce significant levels of 
endo-siRNAs in wild type ovaries [18,32,36,37]. In Su(Hw) mutants, $\mathrm{P}\{$ SUPor-P\} transgenes become part of the endogenous piRNA clusters, producing both pi- and endo-siRNAs.

Previously, it was reported that $\mathrm{P}\{\mathrm{A} \mathrm{ArB}\}, \mathrm{pW}$-hsp-pA and P\{EPgy2 $\}$ transgenes lacking gypsy insulators inserted in Drosophila subtelomeric and telomeric piRNA clusters are incorporated in piRNA production and acquire chromatin properties of their surrounding regions [16,18,32]. Similar to transgenes, TE insertions into the germline piRNA clusters result in the production of cognate piRNAs ensuring silencing of mobilized TEs [15]. We show here, that $\mathrm{Su}(\mathrm{Hw})$-mediated gypsy insulators prevent piRNA production from $\mathrm{P}\{\mathrm{SU}$ Por-P $\}$ transgenic sequences, even if the latter are inserted into endogenous piRNA-producing regions.

\subsection{The Su(Hw) Complex Prevents the Assembly of the Chromatin Structure and Read-Through Transcription Typical of piRNA Clusters}

To learn more about the mechanism of the $\mathrm{Su}(\mathrm{Hw})$-mediated prevention of piRNA production, we compared the chromatin state of transgenes in the wild type and $\mathrm{Su}(\mathrm{Hw})$-mutant backgrounds. In our experiments, we used transheterozygous flies with two $\mathrm{Su}(\mathrm{Hw})$ alleles: the $\mathrm{Su}(\mathrm{Hw})^{\mathrm{V}}$ null allele and the $\mathrm{Su}(\mathrm{Hw})^{\mathrm{f}}$, which carry a defective zinc finger 10 [34]. Su(Hw) ${ }^{\mathrm{f}}$ protein demonstrated the loss of binding to gypsy insulator and the reduced occupancy of many non-gypsy Su(Hw)-binding sites $[34,38]$. ChIP data obtained using anti-Su(Hw) antibodies demonstrate that $\mathrm{P}\{\mathrm{SUPor}-\mathrm{P}\}$ transgenes binds $\mathrm{Su}(\mathrm{Hw})$ in wild type ovaries (Figure 2B). ChIP performed with $s u(H w)^{V} / s u(H w)^{f}$ ovaries, shows a dramatic decrease of $\mathrm{Su}(\mathrm{Hw})$ binding to the gypsy insulator and $\mathrm{P}\{\mathrm{SUPor}-\mathrm{P}\}$ transgenes. As it was reported previously, mutant $\mathrm{Su}(\mathrm{Hw})^{\mathrm{f}}$ is retained at some insulators including site located in $62 \mathrm{D}$ locus $[33,34]$. Indeed, $\mathrm{ChIP}$ demonstrated that mutant $\mathrm{Su}(\mathrm{Hw})$ bound this region, that serves as a positive control (Figure 2B).

The data from ChIP using Rhi, HP1 and H3K9me3 antibodies show that P\{SUPor-P $\}$ transgenes inserted in both the $42 A B$ locus and telomeric retroelement $H e T-A$ lack these chromatin hallmarks in the presence of $\mathrm{Su}(\mathrm{Hw})$ protein (in the wild type genetic background) but acquire them in $\mathrm{Su}(\mathrm{Hw})$-mutant ovaries (Figure $2 \mathrm{C}-\mathrm{E}$ ). At the same time, Rhi binding to $3^{\prime}$ P-element region, located $\sim 5 \mathrm{~kb}$ apart from the gypsy insulators, is not affected by $\mathrm{Su}(\mathrm{Hw})$ mutation in KG09351 and only 1.5-fold increases in $K G 10047 ; s u(H w)^{V / f}$ ovaries (Figure 2C, p3 primer pair). Chromatin of the telomeric element HeT-A and dual-strand piRNA clusters ( $42 A B$ and cluster \#6) was not affected by $\mathrm{Su}(\mathrm{Hw})$ mutation (Figure 2). Therefore, the gypsy insulator complex is established upstream of the Piwi-dependent chromatin formation of piRNA clusters and $\mathrm{Su}(\mathrm{Hw})$ binding mostly affects local chromatin conformation.

Our data suggest that $\mathrm{Su}(\mathrm{Hw})$ binding to $\mathrm{P}\{\mathrm{SUP}$ - $\mathrm{P}-\mathrm{P}\}$ transgenes inserted into piRNA clusters should block transcription of long piRNA precursors. To verify this suggestion, we compared expression of mini-white gene of the transgenes inserted into HeT-A (KG10047 strain) and 42AB (KG09351 strain) in the wild-type and mutant background using transgene-specific primers (Figure 3A). It should be noted that the white promoter shows a very low activity in ovaries. In contrast, we observed that the level of mini-white transcripts was significantly increased in $\mathrm{Su}(\mathrm{Hw})$ mutants for both insertions (Figure 3A). In addition, $\mathrm{Su}(\mathrm{Hw})$ mutations lead to the increased transcript levels of $3^{\prime} \mathrm{P}$ transgenic regions located $5 \mathrm{~kb}$ downstream $\mathrm{Su}(\mathrm{Hw})$ binding sites (Figure $3 \mathrm{~A})$. Strand-specific RT-PCR analysis of 3'P transcription in KG09351 strain demonstrated a lowered level of only sense transgenic transcripts suggesting that the effect of gypsy insulator on transcription is strand-specific (Figure 3B). We therefore suggest that the $\mathrm{Su}(\mathrm{Hw})$-insulator complex should directly interfere with transcription of piRNA clusters by blocking read-through transcription of piRNA precursors. To test this, we conducted RT-PCR using the primers corresponding to the upstream and downstream regions of the transgenic $\mathrm{Su}(\mathrm{Hw})$ site (Figure 2A, p5 primer pair). Accordingly, we revealed transgenic transcripts only in the ovaries of $\mathrm{Su}(\mathrm{Hw})$ mutants (Figure $3 \mathrm{C}$ ). These data indicate that the insulator complex blocks the read-through transcription of transgenic piRNA precursors. Taken together, our data suggest that insertions of the transgenes containing gypsy insulators into endogenous piRNA clusters affect local chromatin conformation, causing the disruption of long piRNA precursor transcription and that this 
effect is mediated by $\mathrm{Su}(\mathrm{Hw})$ binding (Figure 3D). However, the function of the gypsy insulator also requires Centrosomal Protein $190 \mathrm{kD}$ (CP190) and Modifier of mdg4 (Mod67.2) [39,40], the insulator proteins which were not considered here. Therefore, strictly speaking, we could not unambiguously conclude whether or not the effect of $\mathrm{Su}(\mathrm{Hw})$ binding to piRNA clusters demonstrated here was dependent on the entire insulator complex assembly.
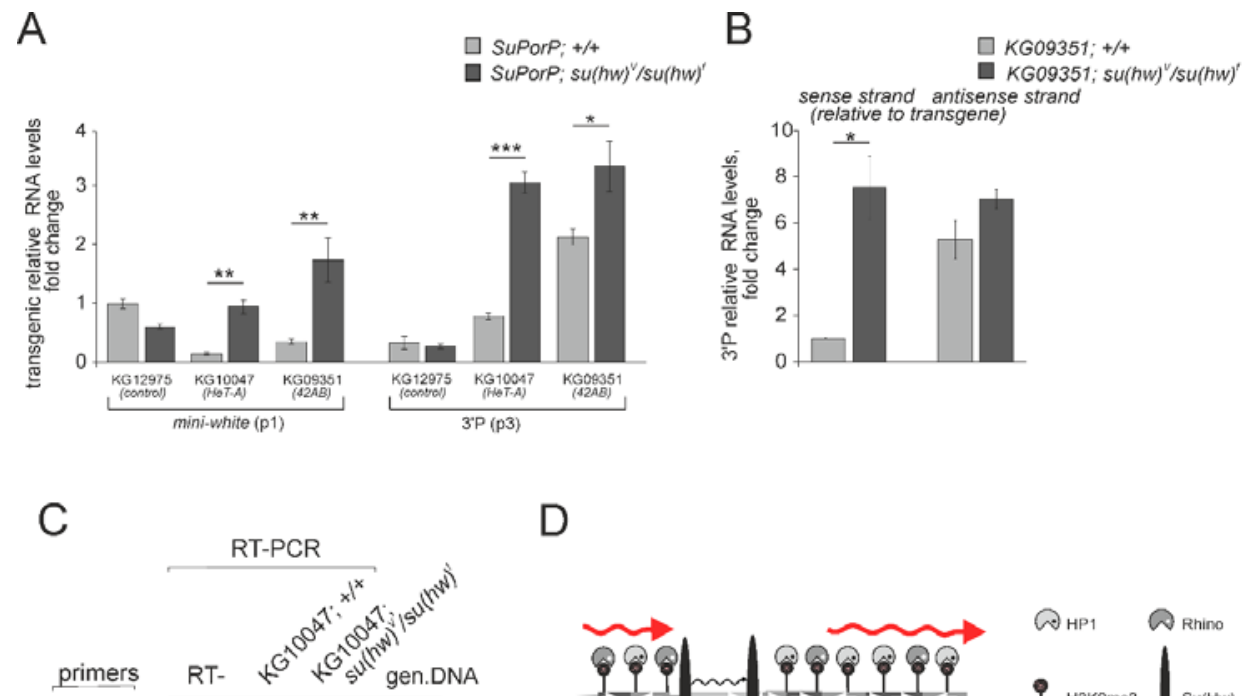

p5
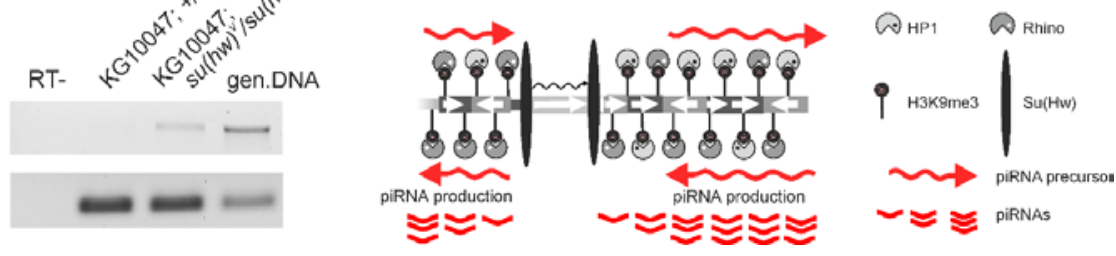

Figure 3. Transcription state of the transgenes containing $\mathrm{Su}(\mathrm{Hw})$ sites and located within piRNA clusters. The positions of primers are indicated in Figure 2A. (A) RT-qPCR analysis of transgenic mini-white and $3^{\prime} \mathrm{P}$ transcripts in the ovaries of transgenic strains in wild type and $\mathrm{Su}(\mathrm{Hw})$ mutant backgrounds. The $\mathrm{P} 1$ primer pair used for RT-PCR specifically detects unspliced transgenic mini-white transcripts. (B) Strand-specific RT-PCR using the $\mathrm{P} 3$ primers corresponding to the transgenic $3^{\prime} \mathrm{P}$ region showed a decreased level of RNA from sense but not from antisense transgenic strand downstream of the $\mathrm{Su}(\mathrm{Hw})$ binding sites. Asterisks indicate statistically significant differences in the expression levels between wild type and $s u(H w)^{V} / s u(H w)^{f}$ mutants $\left({ }^{*} p<0.05\right.$ to $0.01,{ }^{* *} p<0.01$ to $0.001,{ }^{* * *} p<0.001$, unpaired $t$-test). (C) Agarose gel electrophoresis of RT-PCR products shows the presence of read-through transgenic transcripts comprising $\mathrm{Su}(\mathrm{Hw})$-binding sites only in $\mathrm{Su}(\mathrm{Hw})$ mutants. Samples without reverse transcriptase were used as $\mathrm{RT}^{-}$controls. PCR on genomic DNA served as a positive control. (D) Scheme showing that insertion of the $\mathrm{Su}(\mathrm{Hw})$ insulator into the piRNA cluster disrupts local transcription of piRNA precursors, production of small RNAs and formation of specific chromatin structure.

Given the functional integrity of piRNA clusters, we suggested that gypsy insulators might impair the functioning of the cluster regions in the close vicinity of transgenic insertion. To verify this suggestion, we estimated piRNA production in $42 A B$ regions located around the KG09351 insertion.

Due to the presence of highly degenerated TE fragments, piRNA clusters produce abundant piRNAs uniquely mapped to the genome, allowing their mapping to repeat-rich regions [2]. We found that the amount of piRNAs uniquely mapped to the 10-kb region flanking the $3^{\prime}$-end of the transgene was dramatically lower in the presence of the transgene insertion than in the native $42 A B$ locus (Figure $4 \mathrm{~A}$ ).

However, $\mathrm{Su}(\mathrm{Hw})$ mutations restored the level of transgene-flanking piRNAs in the $42 A B$ locus up to the level observed in the native $42 A B$ region in KG10047 strain (Figure 4A). We also show that the production of piRNAs derived from the same region is not affected by $\mathrm{Su}(\mathrm{Hw})$ mutations in KG10047 strain. In addition, the transgenic insertion in $42 A B$ leads to a reduction in transcript levels downstream of the transgene (Figure 4B, transgene is located in the minus genomic strand). Strand-specific RT-PCR analysis using single-mapped primers [41] demonstrated a dramatic decrease in the level of transcripts 
from the negative genomic strand downstream the transgene (Figure 4C). This fact is in agreement with previous result, demonstrating the lowered levels of transgenic $3^{\prime} \mathrm{P}$ sense transcripts (corresponding to the minus genomic strand) in the ovaries of KG09351 strain (Figure 3B). Thus, the $\mathrm{Su}(\mathrm{Hw}$ ) insulator blocks piRNA precursor transcription at least within a 10-kb neighbouring region. Our observations suggest that transgenes containing $\mathrm{Su}(\mathrm{Hw})$-binding sites disrupt long transcription units within piRNA clusters and that this effect is strand-specific. Surprisingly, transgene insertion resulting in lowered level of only antisense transcripts led to the decreased level of both sense and antisense piRNAs uniquely mapped to transgene flanking region in $42 A B$ (Figure $4 \mathrm{~A}$ ). This fact can be explained by an impaired efficiency of ping-pong amplification between piRNA precursors derived from this region. Indeed, it was reported that sense and antisense transcripts originated from the heterochromatic piRNA cluster are involved in the reciprocal cleavage in the course of ping-pong piRNA amplification [42]. Nevertheless, the total abundance of I-specific piRNAs is not affected by the KG09351 insertion in 42AB (Figure S1) because numerous active I-element copies participate in piRNA production in this strain.
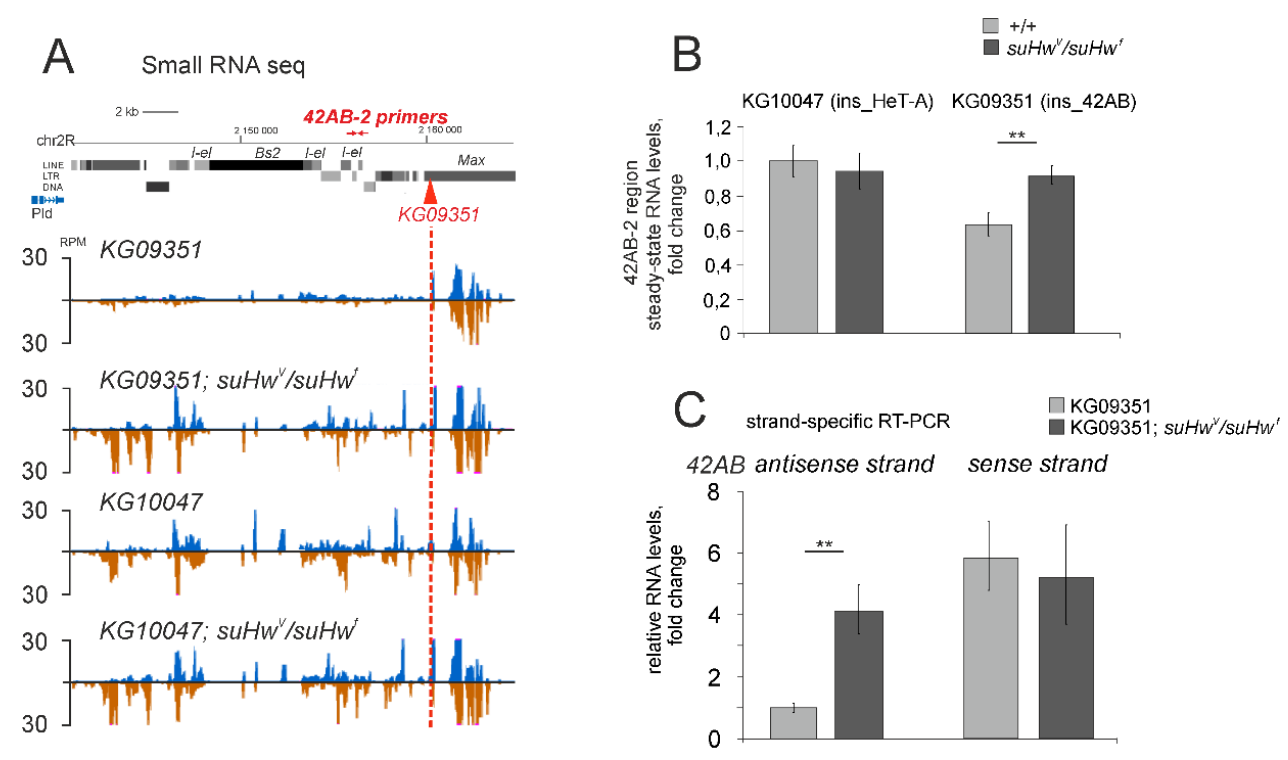

Figure 4. $\mathrm{Su}(\mathrm{Hw})$-binding sites disrupt the integrity of the endogenous piRNA cluster. (A) The effect of SUPor-P transgene inserted in the $42 A B$ locus on piRNA expression. Profile of ovarian small RNA density at the $42 A B$ region adjacent to the KG09351 transgene in wild type and $s u(H w)^{V} / s u(H w)^{f}$ mutant flies and in the KG10047 strain without a transgene insertion in this region. The position of the transgene in the minus genomic strand is designated by a red rectangle. Genomic coordinates are indicated according to the dm6 genome assembly. (B) RT-qPCR analysis of the expression levels of the $42 A B$ region located $4 \mathrm{~kb}$ downstream of the KG09351 transgene. The positions of the primers used for RT-PCR are schematically indicated in (A). (C) Strand-specific RT-PCR using the primers indicated in (A) showed a decreased level of RNA from the negative genomic strand downstream of the transgene insertion. Asterisks indicate statistically significant differences in the expression levels between wild type and $s u(H w)^{V} / s u(H w)^{f}$ mutants $\left(^{*} p<0.05\right.$ to 0.01 , ${ }^{* *} p<0.01$ to 0.001 , ${ }^{* * *} p<0.001$, unpaired $t$-test).

Taken together, these data explain why $\mathrm{Su}(\mathrm{Hw})$ binding results in a local decrease in small RNA production not only from the mini-white located between the insulator sequences but also from the transgenic and genomic flanking regions.

We could not perform the analysis of flanking piRNAs near the KG10047 transgene inserted in the HeT-A $3^{\prime} \mathrm{UTR}$ at $2 \mathrm{R}$ telomere because the unique mapping of small RNAs to poorly assembled and highly repetitive telomeric regions was technically impossible.

To a certain extent, insertion of P\{SUPor-P\} transgene in $42 A B$ was helpful for understanding of the transcription regulation of the major uni-strand flamenco (flam) locus that controls TE expression in ovarian follicular cells $[43,44]$. In contrast to the germline dual-strand piRNA clusters that generate piRNAs 
corresponding to both genomic strands, the flam locus produces primary piRNAs from single strand precursors [2]. Insulator-harbouring TEs, such as gypsy, ZAM and Idefix [22-24], are exceptionally arranged in antisense orientation relative to flam transcription. It is believed that piRNAs complementary to the coding transcripts of these TEs are produced from the single strand precursors transcribed by the flam sense strand [2]. Apparently, gypsy, Zam and Idefix insulators do not interfere with the transcription of the flam piRNA precursors, likely due to a strand-specific mode of insulator influence on transcription.

\section{3. $\mathrm{Su}(\mathrm{Hw})$ Restricts piRNA Production from Telomeric TART Retrotransposons}

The main structural telomeric element, $\operatorname{HeT}-A$, is a non-autonomous retroelement and reverse transcriptase (RT) activity is likely to be provided by TART or TAHRE telomeric retrotransposons. In Drosophila germline, telomeric regions are organized in the piRNA clusters, although telomeric elements are heterogeneous in piRNA production and Rhi binding: TART retrotransposons are less susceptible to piRNA production and Rhi deposition than HeT-A [32]. This implies that TART transcripts may be protected by an unknown mechanism from piRNA biogenesis machinery to provide stable expression of RT essential for telomere elongation in the germline. Here, we present the data suggesting a role for $\mathrm{Su}(\mathrm{Hw})$ in this mechanism.

Using small RNAseq data, we studied the genome-wide impact of $\mathrm{Su}(\mathrm{Hw})$ depletion on transposon-derived piRNA production. The mapping of small RNAs to a canonical set of TEs does not demonstrate global changes caused by $\mathrm{Su}(\mathrm{Hw})$ mutations (Figure 5A, Figure S2).

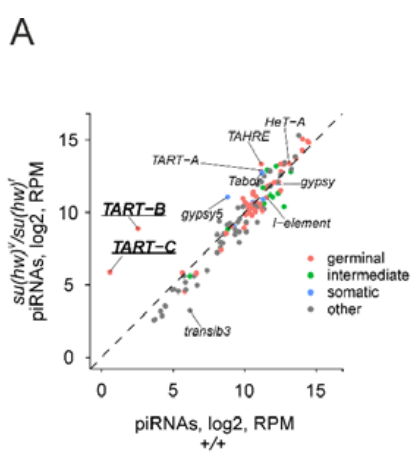

C

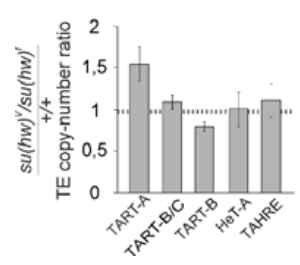

B

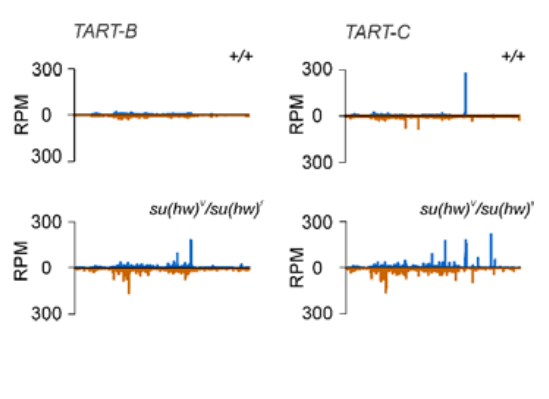

E

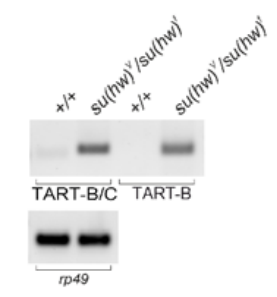

Figure 5. $\mathrm{Su}(\mathrm{Hw})$ depletion leads to the increased production of TART piRNAs. (A) Scatter plots of $\log 2$-transformed and RPM-normalized small RNAseq reads in the ovaries of wild type and $s u(H w)$ mutant flies mapped to the canonical TE sequences. The colour of the dots indicates the type of TEs according to their capacity for maternal deposition in embryos according to [45]. (B) Small RNA mapping to canonical TART-B and TART-C telomeric retrotransposons. Reads mapped to the sense strand are shown in blue and antisense in brown. Analysis of ovarian small RNA libraries from KG10047;+/+ and $K G 10047 ; s u(H w)^{V} / s u(H w)^{f}$ strains (0-3 mismatches allowed) is shown (A,B). (C) qPCR on the genomic DNA was done to estimate relative copy number of telomeric retrotransposons in $K G 10047 ; s u(H w)^{V} / s u(H w)^{f}$ compared to $K G 10047 ;+/+$ strain. Normalization to the single-copy $r p 49$ gene was done. (D) RT-qPCR analysis of transcript levels of TART-A, HeT-A and TAHRE telomeric elements normalized to $r p 49$ in the ovaries. Shown are fold changes of steady-state RNA levels in KG10047; su(Hw) $/ s u(H w)^{f}$ compared to KG10047;+/+ strain. (E) Agarose gel electrophoresis of RT-PCR products demonstrates increased levels of TART-B and TART-C transcripts in $\mathrm{Su}(\mathrm{Hw})$ mutants. $\mathrm{Rp} 49$ is used as a loading control. 
However, piRNA production from few TEs was strongly affected (Table S2). In particular, $\mathrm{Su}(\mathrm{Hw})$ mutations caused a 100-fold increase in the abundance of piRNAs specific to TART-B and TART-C subfamilies of telomeric retrotransposons (Figure 5B, Table S2). To exclude the influence of copy number polymorphism, we evaluated the relative copy number of telomeric retrotransposons in $K G 10047 ;+/+$ and $K G 10047 ; s u(H w)^{V} / s u(H w)^{f}$ strains. To this end, we performed PCR on genomic DNA and showed that the relative copy numbers of HeT-A, TAHRE, TART-A, TART-B and TART-C are very similar in both strains (Figure 5C). These data strongly suggest that TART-B/TART-C transcripts are protected by $\mathrm{Su}(\mathrm{Hw})$-dependent border elements from the piRNA production. Next, we examined the RNA levels of telomeric retroelements in the ovaries of $\mathrm{Su}(\mathrm{Hw})$ mutants by RT-qPCR. We found an increased level of TART-A transcripts in the ovaries of $\mathrm{Su}(\mathrm{Hw})$ mutants, while HeT-A and TAHRE expression was not affected (Figure 5D). However, the levels of TART- $B$ and TART-C transcripts in the wild type ovaries were undetectable by RT-qPCR. Probably, TART expression is limited by a time window during oogenesis, resulting in low levels of TART RNA in the total ovarian RNA. Then, we performed semiquantitative RT-PCR and observed that TART-B and TART-C transcripts were barely revealed in the wild type ovaries but readily detected in the ovaries of $\mathrm{Su}(\mathrm{Hw})$ mutants (Figure $5 \mathrm{E}$ ). These data suggest that $\mathrm{Su}(\mathrm{Hw})$-dependent insulators provide appropriate levels of TART-encoding transcripts in telomeres. $\mathrm{Su}(\mathrm{Hw})$ serves as a transcriptional repressor of coding genes in the ovary [26]. Notably, in wild-type ovaries, TART transcripts are less abundant than upon $\mathrm{Su}(\mathrm{Hw})$ depletion. Thus, $\mathrm{Su}(\mathrm{Hw})$ likely mediates transcriptional repression of TART in the germline.

\subsection{Su(Hw)-Binding Sites Are Depleted from Dual-Strand piRNA Clusters}

What could be happened if the insulator is inserted into the piRNA cluster? To some extent, this situation is simulated by the insertion of $\mathrm{P}\{\mathrm{SUPor}-\mathrm{P}\}$ transgene into the $42 A B$ cluster (Figure 4). Interestingly, the region of the KG09351 insertion in $42 A B$ harbours remnants of ancestral $I$-related retrotransposon, producing abundant piRNAs playing a key role in the control of $I$-element activity [41,46]. The integration of insulator-containing TE nearby I-element fragments in $42 A B$ would strongly decrease the abundance of piRNAs derived from this region and the resistance to I-element mobilization in the strain. One may suggest that shaping endogenous piRNA clusters should be under the constraint of adaptive evolution and, therefore, the germline-specific piRNA clusters should be depleted of insulator-binding sites. To examine this idea, we estimated the average density of $\mathrm{Su}(\mathrm{Hw})$-binding sites in the whole genome and in piRNA clusters (see Materials and Methods). We found that the $\mathrm{Su}(\mathrm{Hw})$ site density was lower in piRNA clusters (one site per $45 \mathrm{~kb}$ ) than in the genome (one site per $14 \mathrm{~kb}, p$-value $<1 \times 10^{-5}$ ). It is tempting to speculate that the spectrum of regulatory sequences associated with TEs in piRNA clusters is subjected to strict selection to provide functional integrity of piRNA-producing loci.

Indeed, mapping of small RNAseq reads to the annotated piRNA clusters [2] did not reveal global changes in the abundance of pi- and endo-siRNAs caused by $\mathrm{Su}(\mathrm{Hw})$ mutations (Figure 6A, Figure S3). Of those affected are many piRNA clusters related to telomeric regions which contain TART and TAHRE retrotransposons (Table S2). However, motif search analyses with the same parameters as above $\left(p\right.$-value $\left.<1 \times 10^{-5}\right)$ failed to identify the $\mathrm{Su}(\mathrm{Hw})$ binding sites in canonical copies of telomeric retrotransposons. Thus, the nature of $\mathrm{Su}(\mathrm{Hw})$ binding sites in telomeres is still to be determined. 
A

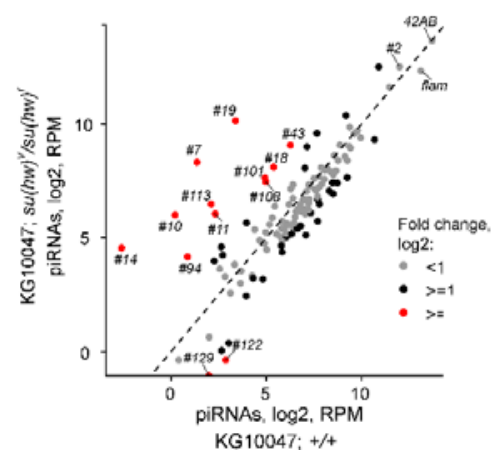

B

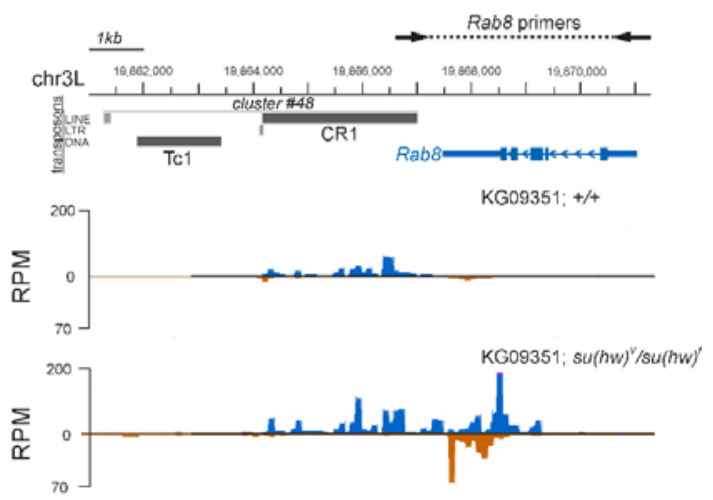

Figure 6. $\mathrm{Su}(\mathrm{Hw})$ depletion leads to increased production of piRNAs in distinct genomic sites. (A) Scatter plot of log2-transformed and RPM-normalized small RNAseq reads in the ovaries of wild type and $s u(H w)^{V} / s u(H w)^{f}$ mutant flies mapped to piRNA clusters (B) Profile of the ovarian small RNA density at the Rab8 gene region located in close proximity to cluster \#48, comprising of Tc1 and CR1 TEs, in wild type and $s u(H w)^{V} / s u(H w)^{f}$ mutant flies. The increased production of small RNAs by both genomic strands in the $\mathrm{Su}(\mathrm{Hw})$ mutant background is shown. Small RNAseq data from the ovaries of $K G 10047 ;+++$ and $K G 10047 ; s u(H w)^{V} / s u(H w)^{f}$ strains were used in the figure. Genomic coordinates are indicated according to the $\mathrm{dm} 6$ genome assembly. PCR on genomic DNA using indicated primers did not reveal variations in the length of the Rab8 gene region.

\subsection{The Su(Hw) Complex Protects Coding Genes from Spurious piRNA Production in the Germline}

Although the loss of $\mathrm{Su}(\mathrm{Hw})$ causes female sterility, its particular role in the female germline development is not well understood $[34,38]$. $\mathrm{Su}(\mathrm{Hw})$ mutations lead to the increased expression of many target genes in the ovary, suggesting that $\mathrm{Su}(\mathrm{Hw})$ serves as a transcriptional repressor during oogenesis [26].

The genome-wide analysis of small RNAseq data demonstrated additional functions of $\mathrm{Su}(\mathrm{Hw})$ in the germline. We revealed a genome-wide effect of $S u(H w)^{V} / S u(H w)^{f}$ mutations on the abundance of piRNAs derived from coding genes (Table S3). Most of the affected genes do not produce piRNAs in a wild-type ovary. The piRNAs corresponding to these genes were observed in $\mathrm{Su}(\mathrm{Hw})^{V} / \mathrm{Su}(\mathrm{Hw})^{f}$ ovaries, suggesting that $\mathrm{Su}(\mathrm{Hw})$ might prevent the spread of piRNA production from piRNA clusters/TEs to neighbouring genes. Indeed, $\mathrm{Su}(\mathrm{Hw})$ depletion causes the appearance of piRNAs antisense to the transcripts of the Rab8 gene located next to the cluster \#48 (Figure 6B). PCR by using genomic DNA did not reveal variations in the length of the gene region producing genic piRNAs, rejecting the possibility of transposon insertion polymorphism. Thus, the insulator most likely blocks transcription of long piRNA precursors, thus protecting coding gene transcripts from entering the piRNA biogenesis machinery in the germline. However, annotated TEs were found in the close vicinity of the affected genes only in a few cases. Surprisingly, we revealed an effect of $\mathrm{Su}(\mathrm{Hw})$ mutations on the abundance of the piRNAs derived from dozens of the coding genes located far from annotated TEs or piRNA clusters (Table S3). At least partly, genic piRNA production may be explained by strain-specific transposon insertions [47]. In most cases, the molecular mechanisms responsible for generation of genic piRNAs in the ovaries of $\mathrm{Su}(\mathrm{Hw})$ mutants remain unclear. It is tempting to speculate that $\mathrm{Su}(\mathrm{Hw})$-mediated complexes perform a barrier function to protect coding gene transcripts from spurious piRNA production in the germline.

\section{Conclusions}

Genomic regions containing damaged transposon fragments were for a long time considered as waste dumps. Most of these regions are piRNA-producing loci that play an essential role in antitransposon defence. The complex regulation of piRNA clusters has evolved to provide piRNA production from the entire piRNA cluster. This mechanism ensures an adaptive response to 
insertions of alien transposons in a piRNA cluster. We show that $\mathrm{Su}(\mathrm{Hw})$ binding sites disrupt the integrity of endogenous piRNA clusters, indicating that the assembly of insulator complexes occurs upstream of cluster-specific chromatin formation. Considering that distinct TE families comprise insulator-binding sites and other regulatory sequences, the TE content of the piRNA clusters should be under strict evolutionary constraints. Moreover, $\mathrm{Su}(\mathrm{Hw})$-mediated complexes likely protect telomeric retrotransposon TART and coding gene transcripts from spurious piRNA production in the germline.

Supplementary Materials: The following are available online at http:/ /www.mdpi.com/2073-4425/10/3/209/s1, Figure S1: Small RNA mapping to the canonical I-element. Figure S2: Genome-wide analysis of Su(Hw) depletion on TE piRNA production (related to Figure 5). Figure S3: Genome-wide analysis of Su(Hw) depletion on small RNA production from piRNA clusters and genes (related to Figure 6). Table S1. Primers used in the study $\left(5^{\prime}\right.$-to-3'). Table S2. Su(Hw) mutations affect piRNA production from some TEs and piRNA clusters. Table S3. $\mathrm{Su}(\mathrm{Hw})$ mutations cause accumulation of genic piRNAs.

Author Contributions: A.K. conceived and designed the experiments; E.R., O.S. and P.A.K. performed the experiments; Y.A. performed genetic experiments; S.R. and P.A.K. performed bioinformatic analysis; A.K. wrote the paper.

Funding: This work was supported by the Russian Science Foundation (grant no. 16-14-10167 to A.K.) and the Russian Foundation for Basic Researches (grant no. 18-34-00415 to E.R.).

Acknowledgments: We thank J. Mason for KG10047 strain, M. Savitsky for helpful advice on genetic crosses, A. Golovnin for $\mathrm{Su}(\mathrm{Hw})$ mutant strains and anti-Su(Hw) antibodies, N. Akulenko for assistance with small RNA library preparation. We also thank the Bloomington Stock Centre for fly strains and the Developmental Studies Hybridoma bank for antibodies.

Conflicts of Interest: The authors declare no conflict of interest.

\section{References}

1. Iwasaki, Y.W.; Siomi, M.C.; Siomi, H. PIWI-Interacting RNA: Its Biogenesis and Functions. Annu. Rev. Biochem. 2015, 84, 405-433. [CrossRef] [PubMed]

2. Brennecke, J.; Aravin, A.A.; Stark, A.; Dus, M.; Kellis, M.; Sachidanandam, R.; Hannon, G.J. Discrete small RNA-generating loci as master regulators of transposon activity in Drosophila. Cell 2007, 128, 1089-1103. [CrossRef] [PubMed]

3. Klattenhoff, C.; Xi, H.; Li, C.; Lee, S.; Xu, J.; Khurana, J.S.; Zhang, F.; Schultz, N.; Koppetsch, B.S.; Nowosielska, A.; et al. The Drosophila HP1 homolog Rhino is required for transposon silencing and piRNA production by dual-strand clusters. Cell 2009, 138, 1137-1149. [CrossRef] [PubMed]

4. Mohn, F.; Sienski, G.; Handler, D.; Brennecke, J. The rhino-deadlock-cutoff complex licenses noncanonical transcription of dual-strand piRNA clusters in Drosophila. Cell 2014, 157, 1364-1379. [CrossRef] [PubMed]

5. Pane, A.; Jiang, P.; Zhao, D.Y.; Singh, M.; Schupbach, T. The Cutoff protein regulates piRNA cluster expression and piRNA production in the Drosophila germline. Embo J. 2011, 30, 4601-4615. [CrossRef] [PubMed]

6. Andersen, P.R.; Tirian, L.; Vunjak, M.; Brennecke, J. A heterochromatin-dependent transcription machinery drives piRNA expression. Nature 2017, 549, 54-59. [CrossRef] [PubMed]

7. Akulenko, N.; Ryazansky, S.; Morgunova, V.; Komarov, P.A.; Olovnikov, I.; Vaury, C.; Jensen, S.; Kalmykova, A. Transcriptional and chromatin changes accompanying de novo formation of transgenic piRNA clusters. RNA 2018, 24, 574-584. [CrossRef] [PubMed]

8. Rangan, P.; Malone, C.D.; Navarro, C.; Newbold, S.P.; Hayes, P.S.; Sachidanandam, R.; Hannon, G.J.; Lehmann, R. piRNA Production Requires Heterochromatin Formation in Drosophila. Curr. Biol. 2011, 21, 1373-1379. [CrossRef] [PubMed]

9. Akkouche, A.; Mugat, B.; Barckmann, B.; Varela-Chavez, C.; Li, B.; Raffel, R.; Pelisson, A.; Chambeyron, S. Piwi Is Required during Drosophila Embryogenesis to License Dual-Strand piRNA Clusters for Transposon Repression in Adult Ovaries. Mol. Cell 2017, 66, 411-419 e414. [CrossRef] [PubMed]

10. Chang, T.H.; Mattei, E.; Gainetdinov, I.; Colpan, C.; Weng, Z.; Zamore, P.D. Maelstrom Represses Canonical Polymerase II Transcription within Bi-directional piRNA Clusters in Drosophila melanogaster. Mol. Cell 2018. [CrossRef] [PubMed] 
11. Zhang, Z.; Wang, J.; Schultz, N.; Zhang, F.; Parhad, S.S.; Tu, S.; Vreven, T.; Zamore, P.D.; Weng, Z.; Theurkauf, W.E. The HP1 homolog rhino anchors a nuclear complex that suppresses piRNA precursor splicing. Cell 2014, 157, 1353-1363. [CrossRef] [PubMed]

12. Chen, Y.C.; Stuwe, E.; Luo, Y.; Ninova, M.; Le Thomas, A.; Rozhavskaya, E.; Li, S.; Vempati, S.; Laver, J.D.; Patel, D.J.; et al. Cutoff Suppresses RNA Polymerase II Termination to Ensure Expression of piRNA Precursors. Mol. Cell 2016, 63, 97-109. [CrossRef] [PubMed]

13. Hur, J.K.; Luo, Y.; Moon, S.; Ninova, M.; Marinov, G.K.; Chung, Y.D.; Aravin, A.A. Splicing-independent loading of TREX on nascent RNA is required for efficient expression of dual-strand piRNA clusters in Drosophila. Genes Dev. 2016, 30, 840-855. [CrossRef] [PubMed]

14. Zhang, F.; Wang, J.; Xu, J.; Zhang, Z.; Koppetsch, B.S.; Schultz, N.; Vreven, T.; Meignin, C.; Davis, I.; Zamore, P.D.; et al. UAP56 couples piRNA clusters to the perinuclear transposon silencing machinery. Cell 2012, 151, 871-884. [CrossRef] [PubMed]

15. Khurana, J.S.; Wang, J.; Xu, J.; Koppetsch, B.S.; Thomson, T.C.; Nowosielska, A.; Li, C.; Zamore, P.D.; Weng, Z.; Theurkauf, W.E. Adaptation to P element transposon invasion in Drosophila melanogaster. Cell 2011, 147, 1551-1563. [CrossRef] [PubMed]

16. Muerdter, F.; Olovnikov, I.; Molaro, A.; Rozhkov, N.V.; Czech, B.; Gordon, A.; Hannon, G.J.; Aravin, A.A. Production of artificial piRNAs in flies and mice. RNA 2012, 18, 42-52. [CrossRef] [PubMed]

17. Todeschini, A.L.; Teysset, L.; Delmarre, V.; Ronsseray, S. The epigenetic trans-silencing effect in Drosophila involves maternally-transmitted small RNAs whose production depends on the piRNA pathway and HP1. PLoS ONE 2010, 5, e11032. [CrossRef] [PubMed]

18. Olovnikov, I.; Ryazansky, S.; Shpiz, S.; Lavrov, S.; Abramov, Y.; Vaury, C.; Jensen, S.; Kalmykova, A. De novo piRNA cluster formation in the Drosophila germ line triggered by transgenes containing a transcribed transposon fragment. Nucleic Acids Res. 2013, 41, 5757-5768. [CrossRef] [PubMed]

19. Kyrchanova, O.; Georgiev, P. Chromatin insulators and long-distance interactions in Drosophila. FEBS Lett. 2014, 588, 8-14. [CrossRef] [PubMed]

20. Chetverina, D.; Aoki, T.; Erokhin, M.; Georgiev, P.; Schedl, P. Making connections: Insulators organize eukaryotic chromosomes into independent cis-regulatory networks. Bioessays 2014, 36, 163-172. [CrossRef] [PubMed]

21. Vogelmann, J.; Valeri, A.; Guillou, E.; Cuvier, O.; Nollmann, M. Roles of chromatin insulator proteins in higher-order chromatin organization and transcription regulation. Nucleus 2011, 2, 358-369. [CrossRef] [PubMed]

22. Brasset, E.; Hermant, C.; Jensen, S.; Vaury, C. The Idefix enhancer-blocking insulator also harbors barrier activity. Gene 2010, 450, 25-31. [CrossRef] [PubMed]

23. Minervini, C.F.; Ruggieri, S.; Traversa, M.; D’Aiuto, L.; Marsano, R.M.; Leronni, D.; Centomani, I.; De Giovanni, C.; Viggiano, L. Evidences for insulator activity of the 5'UTR of the Drosophila melanogaster LTR-retrotransposon ZAM. Mol. Genet. Genom. 2010, 283, 503-509. [CrossRef]

24. Geyer, P.K.; Corces, V.G. DNA position-specific repression of transcription by a Drosophila zinc finger protein. Genes Dev. 1992, 6, 1865-1873. [CrossRef] [PubMed]

25. Gdula, D.A.; Gerasimova, T.I.; Corces, V.G. Genetic and molecular analysis of the gypsy chromatin insulator of Drosophila. Proc. Natl. Acad. Sci. USA 1996, 93, 9378-9383. [CrossRef] [PubMed]

26. Soshnev, A.A.; Baxley, R.M.; Manak, J.R.; Tan, K.; Geyer, P.K. The insulator protein Suppressor of Hairy-wing is an essential transcriptional repressor in the Drosophila ovary. Development 2013, 140, 3613-3623. [CrossRef] [PubMed]

27. Biessmann, H.; Prasad, S.; Semeshin, V.F.; Andreyeva, E.N.; Nguyen, Q.; Walter, M.F.; Mason, J.M. Two distinct domains in Drosophila melanogaster telomeres. Genetics 2005, 171, 1767-1777. [CrossRef] [PubMed]

28. Langmead, B.; Salzberg, S.L. Fast gapped-read alignment with Bowtie 2. Nat. Methods 2012, 9, 357-359. [CrossRef] [PubMed]

29. Radion, E.; Ryazansky, S.; Akulenko, N.; Rozovsky, Y.; Kwon, D.; Morgunova, V.; Olovnikov, I.; Kalmykova, A. Telomeric Retrotransposon HeT-A Contains a Bidirectional Promoter that Initiates Divergent Transcription of piRNA Precursors in Drosophila Germline. J. Mol. Biol. 2017, 429, 3280-3289. [CrossRef] [PubMed] 
30. Golovnin, A.; Volkov, I.; Georgiev, P. SUMO conjugation is required for the assembly of Drosophila $\mathrm{Su}(\mathrm{Hw})$ and $\operatorname{Mod}(\operatorname{mdg} 4)$ into insulator bodies that facilitate insulator complex formation. J. Cell Sci. 2012, 125, 2064-2074. [CrossRef] [PubMed]

31. Grant, C.E.; Bailey, T.L.; Noble, W.S. FIMO: Scanning for occurrences of a given motif. Bioinformatics 2011, 27, 1017-1018. [CrossRef] [PubMed]

32. Radion, E.; Morgunova, V.; Ryazansky, S.; Akulenko, N.; Lavrov, S.; Abramov, Y.; Komarov, P.A.; Glukhov, S.I.; Olovnikov, I.; Kalmykova, A. Key role of piRNAs in telomeric chromatin maintenance and telomere nuclear positioning in Drosophila germline. Epigenetics Chromatin 2018, 11, 40. [CrossRef] [PubMed]

33. Melnikova, L.; Kostyuchenko, M.; Parshikov, A.; Georgiev, P.; Golovnin, A. Role of Su(Hw) zinc finger 10 and interaction with $\mathrm{CP} 190$ and $\mathrm{Mod}(\mathrm{mdg} 4)$ proteins in recruiting the $\mathrm{Su}(\mathrm{Hw})$ complex to chromatin sites in Drosophila. PLoS ONE 2018, 13, e0193497. [CrossRef] [PubMed]

34. Baxley, R.M.; Soshnev, A.A.; Koryakov, D.E.; Zhimulev, I.F.; Geyer, P.K. The role of the Suppressor of Hairy-wing insulator protein in Drosophila oogenesis. Dev. Biol. 2011, 356, 398-410. [CrossRef] [PubMed]

35. Gunawardane, L.S.; Saito, K.; Nishida, K.M.; Miyoshi, K.; Kawamura, Y.; Nagami, T.; Siomi, H.; Siomi, M.C. A slicer-mediated mechanism for repeat-associated siRNA $5^{\prime}$ end formation in Drosophila. Science 2007, 315, 1587-1590. [CrossRef] [PubMed]

36. Czech, B.; Malone, C.D.; Zhou, R.; Stark, A.; Schlingeheyde, C.; Dus, M.; Perrimon, N.; Kellis, M.; Wohlschlegel, J.A.; Sachidanandam, R.; et al. An endogenous small interfering RNA pathway in Drosophila. Nature 2008, 453, 798-802. [CrossRef] [PubMed]

37. De Vanssay, A.; Bouge, A.L.; Boivin, A.; Hermant, C.; Teysset, L.; Delmarre, V.; Antoniewski, C.; Ronsseray, S. Paramutation in Drosophila linked to emergence of a piRNA-producing locus. Nature 2012, 490, 112-115. [CrossRef] [PubMed]

38. Soshnev, A.A.; He, B.; Baxley, R.M.; Jiang, N.; Hart, C.M.; Tan, K.; Geyer, P.K. Genome-wide studies of the multi-zinc finger Drosophila Suppressor of Hairy-wing protein in the ovary. Nucleic Acids Res. 2012, 40, 5415-5431. [CrossRef] [PubMed]

39. Georgiev, P.; Kozycina, M. Interaction between mutations in the suppressor of Hairy wing and modifier of mdg4 genes of Drosophila melanogaster affecting the phenotype of gypsy-induced mutations. Genetics 1996, 142, 425-436. [PubMed]

40. Pai, C.Y.; Lei, E.P.; Ghosh, D.; Corces, V.G. The centrosomal protein CP190 is a component of the gypsy chromatin insulator. Mol. Cell 2004, 16, 737-748. [CrossRef] [PubMed]

41. Ryazansky, S.; Radion, E.; Mironova, A.; Akulenko, N.; Abramov, Y.; Morgunova, V.; Kordyukova, M.Y.; Olovnikov, I.; Kalmykova, A. Natural variation of piRNA expression affects immunity to transposable elements. PLoS Genet. 2017, 13, e1006731. [CrossRef] [PubMed]

42. Grentzinger, T.; Armenise, C.; Brun, C.; Mugat, B.; Serrano, V.; Pelisson, A.; Chambeyron, S. piRNA-mediated transgenerational inheritance of an acquired trait. Genome Res. 2012, 22, 1877-1888. [CrossRef] [PubMed]

43. Desset, S.; Meignin, C.; Dastugue, B.; Vaury, C. COM, a heterochromatic locus governing the control of independent endogenous retroviruses from Drosophila melanogaster. Genetics 2003, 164, 501-509. [PubMed]

44. Sarot, E.; Payen-Groschene, G.; Bucheton, A.; Pelisson, A. Evidence for a piwi-dependent RNA silencing of the gypsy endogenous retrovirus by the Drosophila melanogaster flamenco gene. Genetics 2004, 166, 1313-1321. [CrossRef] [PubMed]

45. Malone, C.D.; Brennecke, J.; Dus, M.; Stark, A.; McCombie, W.R.; Sachidanandam, R.; Hannon, G.J. Specialized piRNA pathways act in germline and somatic tissues of the Drosophila ovary. Cell 2009, 137, 522-535. [CrossRef] [PubMed]

46. Brennecke, J.; Malone, C.D.; Aravin, A.A.; Sachidanandam, R.; Stark, A.; Hannon, G.J. An epigenetic role for maternally inherited piRNAs in transposon silencing. Science 2008, 322, 1387-1392. [CrossRef] [PubMed]

47. Shpiz, S.; Ryazansky, S.; Olovnikov, I.; Abramov, Y.; Kalmykova, A. Euchromatic transposon insertions trigger production of novel Pi- and endo-siRNAs at the target sites in the drosophila germline. PLoS Genet. 2014, 10, e1004138. [CrossRef] [PubMed]

(C) 2019 by the authors. Licensee MDPI, Basel, Switzerland. This article is an open access article distributed under the terms and conditions of the Creative Commons Attribution (CC BY) license (http:/ / creativecommons.org/licenses/by/4.0/). 